\title{
A Comparative Study of Dexmedetomidine And Propofol Infusion For Sedation in Intensive Care Unit- A Prospective, Randomised, Double Blinded Study
}

\author{
Arvind Kumar ${ }^{1}$, Chandan Kumar Keshri ${ }^{2}$, Mumtaz Hussain ${ }^{3}$, Shashi Kant, \\ Ritesh Kumar ${ }^{4}$, Rajneesh Kumar ${ }^{5}$,Rupam Kumari ${ }^{6}$.
}

\section{Introduction}

Critically ill patients in the Intensive care unit (ICU) are prone to many adverse clinical situations because of their coexisting disease or the ICU environment that produce harmful psychological and physiological changes. These changes are due to increased levels of catecholamine's and other stress hormones. The critically ill patients in the ICU are subjected to pain and discomfort due to endotracheal intubation and mechanical ventilation, intermittent physiotherapy, tracheal suction etc. Nursing procedures can also be unpleasant for them ${ }^{[1]}$ The noise level produced by the monitoring and support equipments are usually high and irritating and the lighting in the ICU surrounding are not pleasant rather it is unsoothing to the eyes, enhancing the adverse reactions. ${ }^{[2]}$ The goal of sedation in mechanically ventilated patients is to keep them calm and without agitation to maximize patient comfort and ventilator synchrony. ${ }^{[3]}$ An ideal sedative should provide a rapid onset, a rapid recovery, have low profile to accumulate, leaving no withdrawal effects, should be easily titratable and should not disturb or minimal disturb hemodynamic stability and has some analgesic property ${ }^{[4]}$ In addition to careful drug selection by the ICU team, nursing-driven sedation protocols, pharmacist intervention, and scheduled daily interruption of sedation have been shown to improve patient outcomes and decrease overall time on sedation ${ }^{[5-7]}$

Dexmedetomidine is a centrally acting, alpha-2 adrenergic receptor agonist approved in 1999 by the U.S. Food and Drug Administration (FDA) for sedation in mechanically ventilated patients during the first 24 hours and in nonintubated patients prior to and/or during surgical or nonsurgical procedures ${ }^{[8]}$ It is the first and only alpha-2 agonist approved by the FDA for sedation. It is now established as a novel approach to intensive care sedation and has the potential to reshape patient care in the ICU. ${ }^{[9]}$ Dexmedetomidine offers the advantage over Propofol due to its minimal effect on respiration, its analgesic efficacy, and its effect on the hemodynamic. ${ }^{[10,11]}$ Dexmedetomine has shown to be effective for sedation while reducing the incidence of delirium and need for opioids, as dexmedetomidine also has analgesic properties. ${ }^{[12]}$ The most common side effects associated with the use of dexmedetomidine include hypotension (24\%-54\%) and bradycardia (5\%$14 \%$ ), which are concerning in this patient population and may limit dexmedetomidine usage.

Propofol is a sedative-hypnotic agent used in the induction or maintenance of anaesthesia or sedation $^{[13]}$ Due to its rapid onset, short duration of action, and relatively low cost, it has long been use as standard medication for sedation. Severe hemodynamic instability, such as bradycardia and hypotension, both of which commonly occur at standard doses, has been shown to be a use-limiting side effect. ${ }^{[14-17]}$ Propofol is also formulated in a fat-based emulsion that can cause hypertriglyceridemia after prolonged use. ${ }^{[18-19]}$

The present study is designed to compare the quality of sedation between Dexmedetomidine and Propofol infusion in post operative patients admitted in ICU.

Material

The study was carried out in Post-operative patients who were admitted in the ICU of IGIMS Patna. Sixty adult post-operative Patients (ages 18 to 60 years) between ASA grade I to III, who were intubated and mechanically ventilated for a period of approximately twenty four hours were included in the study. After approval by hospital ethical committee, informed consent was obtained from one of patient's close relatives.

The patient who excluded from study are Known or suspected allergy to Dexmedetomidine or Propofol, Severe hepatic or renal disease, Requirement of muscle relaxants except for intubation (succinylcholine), Pregnancy or lactation, Severe pulmonary or cardiac disorder.

Sixty post-operative mechanically ventilated patients after major abdominal or pelvic surgery that required mechanical ventilation in the ICU was selected. Group D - Dexmedetomidine group will receive a loading dose- $1 \mathrm{mcg} / \mathrm{kg}$ and a Maintenance dose- $0.5 \mathrm{mcg} / \mathrm{kg} / \mathrm{hr}$. Group P - Propofol group will receive a loading dose- $1 \mathrm{mg} / \mathrm{kg}$ and a Maintenance dose- $0.5 \mathrm{mg} / \mathrm{kg} / \mathrm{hr}$. Analgesia with morphine bolus doses $0.05 \mathrm{mg} / \mathrm{kg}$ body weight was administered as per need. The efficacy of sedation was assessed by using the Ramsay sedation score. 
Three levels of sedation were considered:

(1) Adequate, when the sedation level was grade 2, 3, 4 or 5 on Ramsay scale. (2) Insufficient when the sedation level was grade 1 on Ramsay scale.

(3) Excessive, when the sedation level was grade 6 on Ramsay scale.

The aim of our study was to achieve a target sedation of grade 3 on Ramsay scale for most of the sedation hours.

The aim of our study was to have a comfortable sedated patient with no pain at rest and minimal pain at movement. Pain was considered as the $1^{\text {st }}$ cause of inadequate analgesia/sedation and was treated first with low dose of morphine before further increasing the dose of Dexmedetomidine or Propofol.

At the end of each shift, the nurse attending the patient was interviewed regarding the efficacy and overall quality of sedation. If patients considered fit for weaning from mechanical ventilation, weaning was started while the patients were still sedated.

Weaning was attempted when arterial oxygen tension was $75 \mathrm{mmHg}$ or more with an inspired oxygen concentration of $40 \%$ or less. When considered clinically appropriate, the patient was disconnected from ventilator and oxygen enriched air was provided by T-piece.

The following parameters were monitored during the study: - ECG, HR, SBP, MAP, CVP, SPO ${ }_{2}, \mathrm{RR}, \mathrm{VT}, \mathrm{ABG}$ and Ramsay sedation score.

Data were expressed as mean $\pm \mathrm{SD}$. Group means were compared using student's t-test. A 'P' value of $<0.05$ was considered statistically significant.

\section{Results}

Sixty patients were entered into this study. Thirty patients were in Dexmedetomidine group and thirty in Propofol group.

The mean age in Dexmedetomidine groups was $47.6 \pm 12.41$ yrs. and that in Propofol group was $40.87 \pm 15.70$ yrs. The mean weight was $54.60 \pm 6.74$ and $55.07 \pm 6.11 \mathrm{~kg}$ respectively. The male to female ratio was 22:8 in Dexmedetomidine group and 20:10 in Propofol group. There was no difference between the two groups with regards to age, weight, sex and ASA grades. The patient population in both the groups were also similar with regards to the type of surgery done.

Sedative and analgesic requirements

The mean total sedation requirement was $495 \pm 185 \mu \mathrm{g}$ in Dexmedetomidine group and $55.7 \pm 21.7 \mathrm{mg}$ in Propofol group. The mean hourly dose of sedative was $0.34 \pm 0.13 \mu \mathrm{g} / \mathrm{kg} / \mathrm{hr}$. in Dexmedetomidine group and $0.042+0.017 \mathrm{mg} / \mathrm{kg} / \mathrm{hr}$. in Propofol group.

Patients in Propofol group required more number of boluses of analgesics compared to Dexmedetomidine group.

\section{Heart Rate:}

The mean heart rate at the start of infusion was $116.50 \pm 14.26 \mathrm{bpm}$ in Dexmedetomidine group and $115.77 \pm 12.99$ in Propofol group. Following the start of infusion heart rate was lowered in both the groups. The lowest heart rate in both groups was observed at $24 \mathrm{hrs}$. of infusion. At the end of 24 hour, the heart rate in Dexmedetomidine group was 94.17 and that in Propofol group was 107.13.The heart rate was lowered to a greater extent in Dexmedetomidine group than in Propofol group at the end of 24 hour of infusion.

This was however statistically not significant (Graph1).

\section{Systolic Blood Pressure}

The baseline SBP (mmHg) in the Dexmedetomidine and Propofol Group were $132.70 \mathrm{mmHg}$ and $133.80 \mathrm{mmHg}$ respectively. At one hour from start of infusion, the corresponding values were 123.00 and $127.00 \mathrm{mmHg}$ in Dexmedetomidine group and Propofol group respectively. The maximum fall in SBP in Dexmedetomidine group was $118.23 \mathrm{mmHg}$ at 24 hours from start of infusion. The maximum fall in Propofol group was 125.27 at 24 hours of infusion. The fall of SBP in both the groups over the period of study was found to be statistically significant at all-time points from the baseline values, in both the groups (Graph 2).

\section{Mean Arterial Blood pressure:}

The baseline MAP (mmHg) in the Dexmedetomidine and Propofol Group were 109.60 \pm 8.63 and $106.30 \pm 7.10 \mathrm{mmHg}$ respectively. Patient in Dexmedetomidine group had a mean MAP of $103.80 \pm 5.20 \mathrm{mmHg}$ compared to102.63+7.36 observed in the Propofol group at 10 min from start of infusion. At one hour from start of infusion, the corresponding values were 101.57+5.19 and103.27+6.70 mmHg in Dexmedetomidine group and Propofol group respectively. The maximum fall in MAP in Dexmedetomidine group was from baseline values of $109.60+8.63 \mathrm{mmHg}$ to $98.43 \pm 5.56$ $\mathrm{mmHg}$ at 24 hours from start of infusion. The maximum fall in Propofol group was from baseline 
values of $106.30 \pm 7.10 \mathrm{mmHg}$ to $100.27 \mathrm{mmHg}$ at 20 hours of infusion. Throughout the period of study, patients in Dexmedetomidine group maintained a lower MAP than patients in Propofol group at corresponding times. The fall of MAP in both the groups over the period of study was found to be statistically significant at all-time points from the baseline values, in both the groups (Graph

\section{3).Central Venous Pressure:}

The baseline CVP in the Dexmedetomidine and Propofol Groups were $11.13 \pm 1.48$ and $9.80 \pm 1.85$ $\mathrm{mmHg}$ respectively. In both the groups there was a significant fall in CVP with time. The lowest value of CVP was recorded at 24 hours of infusion in Dexmedetomidine group and at 12 hours in Propofol group. The fall of CVP in Dexmedetomidine group over the period of study was found to be statistically significant at all-time points from the baseline values.

\section{Respiratory rate and $\mathrm{SPO}_{2}$ :}

The mean baseline spontaneous respiratory rate at the start of infusion was 21.17 breaths per minute in Dexmedetomidine group and 21.97 breaths per minute in Propofol group. At the end of study, the respiratory rate in Dexmedetomidine group was 18.83 breaths per minute compared to 16.70 breaths per minute in Propofol group. There was minimal change in RR in dexmedetomidine group while in Propofol group changes were slightly more (Graph 4).

All the patients in both groups maintained a percentage saturation of oxygen around $95 \%$ while on spontaneous breathing throughout the study

\section{Quality of Sedation:}

We compared the quality of sedation using Ramsay sedation score. At the start of the study 11 patients in Dexmedetomidine group and 7 patients in Propofol group were agitated (RSS=1). By 1 hour from start of sedation the numbers had decreased to 1 in Dexmedetomidine whereas 3 patients in Propofol group were still agitated. 23 patients in the Dexmedetomidine group had a baseline Ramsay sedation score of less than optimal (RSS=3), compared to 20 patients in Propofol group. By the end of 1 hour from start of infusion, 12 patients in Dexmedetomidine group had achieved the sedation score of 3 in contrast to 11 patients in Propofol group.

However the patients in Dexmedetomidine group remained in excessive sedation on considerably fewer occasions compared to patients in Propofol group. This was considered statistically significant. The patients in Propofol group needed more frequent changes in their infusion rate than patients in Dexmedetomidine group.

Patients in Dexmedetomidine group achieved a target Ramsay score of 3 on statistically more number of occasions compared to patients in Propofol group. However, the total number of observations made within the acceptable sedation score of 2 to 5 was similar between both the groups.

Acceptable: Ramsay sedation score 2-5

Recovery from sedation

The recovery from sedation was significantly rapid in Dexmedetomidine group. Patients in Dexmedetomidine group were easily aroused and gripped observer`s hand earlier after stoppage of sedation. In contrast patients in the Propofol group took a longer time to achieve eye opening on command and gripped observer`s hand.

\section{Discussion}

ICU Sedation is long debated subject as there has been lot of progress in selection of these sedative drugs for ICU use. The updated recommendations from the Society of Critical Care Medicine and the American Society of Health-System Pharmacist Pain, Sedation and Analgesia guidelines, released in 2012, recommend first-line sedation with dexmedetomidine or Propofol for most patients. This study and previous work $^{[20]}$ have shown dexmedetomidine to be an effective and safe agent for use as post-operative sedation in the ICU.

The primary objective of this study was to evaluate the sedation characteristics of Dexmedetomidine and Propofol in postoperative mechanically ventilated patients in our ICU. The study was conducted for a period of 24 hours.

In our study, more number of patients in Dexmedetomidine group achieved the optimal sedation (Ramsay score

3) earlier and remained for a significantly longer time period than patients in Propofol group.

The dose of Dexmedetomidine used in our study was lower than doses used in earlier studies. For short term sedation of postoperative patients, the dose ranged between 0.2 to $0.7 \mu \mathrm{g} / \mathrm{kg} / \mathrm{hr}$. Our mean dose of Propofol was also lesser than the dose used in many earlier studies. In our studies both the groups had a lower requirement, compared to other studies. 
The assessment of the recovery of level of consciousness and weaning time has been favourable to Dexmedetomidine in most studies. We did not assess weaning time and time of extubation in our study, only wake up time and time to perform a simple but specific motor function were assessed. This is because the underlying medical condition of the patients and the nature of the surgery limited their early extubation.

The Dexmedetomidine group in our study had a faster recovery from discontinuation of sedative infusion. In our study, patients in Dexmedetomidine groups were easily aroused and gripped observer's hand earlier than the patients in Propofol group. This difference was found to be statistically significant. The hemodynamic of dexmedetomidine is predictable from the pharmacology of $\alpha_{2}$ adrenoceptor agonists, and has been confirmed from previous studies in volunteers, ${ }^{[21-22]}$ patients under anaesthesia ${ }^{[23-24] \text { and }}$, more recently, ICU patients ${ }^{[.20]}$ In our study both Dexmedetomidine and Propofol had a significant fall from baseline values of systolic and mean arterial pressure, the fall being greater in Dexmedetomidine group.

We observed a lower heart rate in Dexmedetomidine group patients than Propofol group patients. This was consistent with the observations made by other.

Although the cost of Dexmedetomidine was higher in our study, the ease of titrability, optimal sedation and rapid recovery offered by it makes it a superior choice over Propofol. Our use of Ramsay Sedation Score had some limitations. The scale is a compromise between accuracy, simplicity and ease of use.

Conclusion

My study showed comparison of dexmedetomidine and Propofol for sedation in patients after major abdominal or pelvic surgery. In conclusion, dexmedetomidine appears to be a safe and acceptable ICU sedative agent when both the clinician's and patient's perspectives are considered. Weaning from ventilator was rapid and ICU stay is short in patient receiving dexmedetomidine.

\section{Refrences}

[1]. Aitkenhead AR, Pepperman ML, Willatts SM et al. Comparison of propofol and midazolam for sedation of critically ill patients. Lancet 1989; 11:704-709.

[2]. Easton C, McKenzie F. Sensory perceptual alterations in the intensive care unit. Heart Lung 1988; 17:229-35.

[3]. The goal of sedation in mechanically ventilated patients is to keep them calm and without agitation to maximize patient comfort and ventilator synchrony.

[4]. Coursin DB, Coursin DB, Maccioli GA. Dexmedetomidine. Curr Opin Crit care 2001; 7: 221-226. .

[5]. Schweickert WD, Kress JP. Strategies to optimize analgesia and sedation. Critical Care. 2008;12(3).

[6]. Sessler CN, Pedram S. Protocolized and target-based sedation and analgesia in the ICU. Anesthesiol Clin.2011;29(4):625-50.

[7]. Marshall J, Finn CA, Theodore AC. Impact of a clinical pharmacist-enforced intensive care unit sedation protocol on duration of mechanical ventilation and hospital stay. Crit Care Med. 2008;36(2):427-433.

[8]. Precedex [package insert]. Lake Forest, IL: Hospira, Inc; 2010.

[9]. Ebert JT Current strategies in ICU sedation; Dannenmiller Memorial Education Foundation. 12500 Network Blvd, Suite 101, San Antonio, TX. USA.

[10]. Rowe K and Fletcher S. Sedation in ICU. Continuing education in Anaesthesia, critical care \& pain. 2008;8:50-55.

[11]. 11.Purugganan RV. S Afr J AnaesthesiolAnalg.2010;16:116- 120.

[12]. Martin E, Ramsay G, Mantz J, Sum-Ping ST. The role of the alpha2-adrenocepter agonist dexmedetomidine in postsurgical sedation in the intensive care unit. J Intensive Care Med. 2003;18:29-41..

[13]. Diprivan [package insert]. Wilmington DE: AstraZeneca Pharmaceuticals LP;2005

[14]. Roberts RJ, Barletta JF, Fong JF, Schumaker G, Kuper PJ, Papadopoulos S, et al. Incidence of propofol-related infusions syndrome in critically ill adults: a prospective, multicenter study. Critical Care. 2009;13

[15]. Fodale V, La Monaca E. Propofol infusion syndrome: an overview of a perplexing disease. Drug Saf.2008;31(4):293-303.

[16]. Corbett SM, Montoya ID, Moore FA. Propofol-related infusion syndrome in intensive care patients.Pharmacotherapy. 2008;28(2):250-258.

[17]. Marik PE. Propofol: therapeutic indications and side-effects. Curr Pharm Des. 2004;10(29):3639-49.

[18]. Devlin JW, Lau AK, Tanios MA. Propofol-associated hypertriglyceridemia and pancreatitis in the intensive care unit: an analysis of frequency and risk factors. Pharmacotherapy. 2005;25(10):1348-1352.

[19]. Gottschling S, Larsen R, Meyer S, Graf N, Reinhard H. Acute pancreatitis induced by short-term propofol administration. Paediatr Anaesth. 2005;15(11):1006-8..

[20]. 20.Venn R, Bradshaw C, Spencer R, et al. Preliminary UK experience of dexmedetomidine, a novel agent for postoperative sedation in the intensive care unit. Anaesthesia 1999; 54: 1136-42

[21]. Kallio A, Scheinin M, Koulu M, et al. Effects of dexmedetomidine, a selective alpha 2-adrenoceptor agonist, on hemodynamic control mechanisms. Clin Pharmacol Ther 1989; 46: 33-42

[22]. Bloor BC, Ward DS, Belleville JP, Maze M. Effects of intravenous dexmedetomidine in humans. II. Hemodynamic changes. Anesthesiology 1992; 77:1134-42

[23]. Jalonen J, Hynynen M, Kuitunen A, et al. Dexmedetomidine as an anesthetic adjunct in coronary artery bypass grafting. Anesthesiology 1997; 86: 331-45

[24]. Talke P, Li J, Jain U, et al. Effects of perioperative dexmedeto midine infusion in patients undergoing vascular surgery.The Study of Perioperative Ischemia Research Group. Anesthesiology 1995; 82: 620-33 